\title{
Prediction and suppression of beam breakup instability in multicell superconducting cavities
}

\author{
V. Volkov* \\ Budker Institute of Nuclear Physics (BINP), Lavrentiev Avenue, 11, 630090 Novosibirsk, Russia
}

(Received 2 September 2008; published 28 January 2009)

\begin{abstract}
Beam breakup instability in superconducting cavities is a serious problem. In this work, a four-cell LEP cavity installed in the KAERI linear accelerator is considered as an example. Dependence of the breakup instability threshold currents on the characteristics of a dipole mode was determined both analytically and numerically. An efficient technique to suppress breakup instability using rf beam focusing within a cavity is suggested. The technique involves applying TE-type monopole higher-order modes and is useful for multicell superconducting cavities with many trapped high- $Q$ dipole modes.
\end{abstract}

\section{INTRODUCTION}

Experiments with electron beam current in a linear accelerator at KAERI were confronted with the beam breakup instability problem. This accelerator has a $2 \mathrm{MeV}$ electron beam injector and two four-cell superconducting LEP cavities (see Fig. 1) operating at $352 \mathrm{MHz}$. Both the LEP cavities and the injector were installed with the help of BINP scientists [1].

The beam current in the accelerator is incrementally increased by changing the repetition rate of bunches. At an average current of $4.5 \mathrm{~mA}$, the beam becomes unstable and, in a short time, is lost. The breakup is caused by the interaction of the beam with one of the higher-order dipole modes in the cavity. The pickup coupling with the instability acting dipole mode is absent, so detection of this mode was impossible in experiments. It has been found that the threshold current of breakup instability is independent of repetition rate, bunch charge, initial small beam offsets, and slopes [2]. It is important to investigate why the beam current threshold does not depend on these parameters, and whether there is a similar instability for continuous beams.

This paper is organized in the following manner: Sec. II discusses the features of dipole higher-order modes (HOMs) of the LEP cavity calculated up to $2 \mathrm{GHz}$ using CLANS2 code [3]; Sec. III contains the theoretical derivation of the basic equations for beam-dipole-mode interaction and threshold current for breakup instability; Sec. IV discusses the threshold currents for some dipole HOMs, found and calculated using ASTRA code [4]; and Sec. V analyzes rf focusing by a TE HOM to suppress breakup instability in superconducting LEP cavity, the parameters of a TE HOM and the behavior of the breakup instability threshold current versus the $\mathrm{TE}_{021}$ mode are calculated and presented.

*V.N.Volkov@inp.nsk.su

\section{DIPOLE HIGHER-ORDER MODES OF THE LEP CAVITY}

The dipole HOMs of the LEP cavity were calculated using CLANS2 code [3]. The LEP cavity dimensions at room temperature are given in Ref. [5]. All radial dimensions used to model the rf field were decreased by a factor 1.005 to account for the thermal contraction from room temperature to cryogenic temperatures. The longitudinal dimensions were not changed since the cavity length determines the fundamental mode frequency. In this case, all calculated HOM frequencies are $0.5 \%$ to $1 \%$ higher than those measured at room temperature without vacuum in the cavity [6].

In CLANS2 calculations, absorbing loads were installed in the beam pipe to model complete damping of propagating dipole modes and to create trapped modes in the cavity (see Fig. 1). The loads are a lossy material with complex $\mu$ and $\varepsilon$ that match the beam pipes.

The dipole HOMs of an azimuthally symmetric cavity have only transverse electromagnetic fields on axis. The longitudinal fields grow linearly (to first approximation) with the distance from the axis. According to the definition, TE/TM modes do not have concurrent longitudinal electric and magnetic field components like this in a simple pillbox

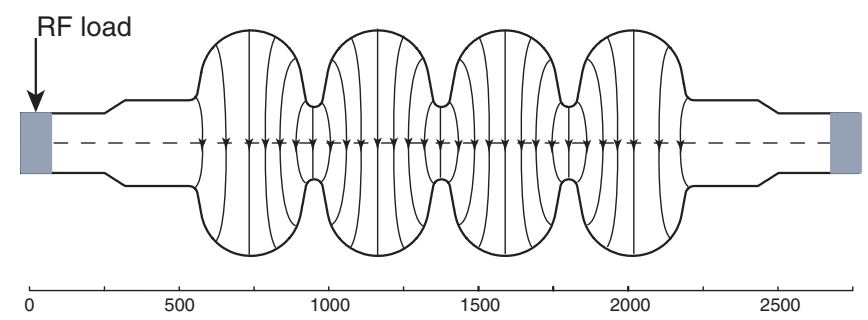

FIG. 1. (Color) A sketch of the LEP cavity. The rf electric field pattern is shown for the dipole mode at $446 \mathrm{MHz}$ with vertical polarization (pillboxlike $\mathrm{TE}_{111}$, 0-type). In calculations, the loads of the beam pipes are matched to dipole modes propagating into the beam pipes. 


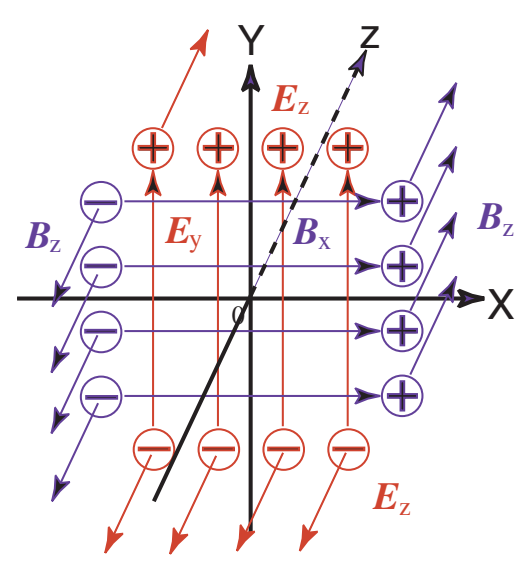

FIG. 2. (Color) Dipole HOM field in a cross section near the cavity axis.

cavity. But beam pipes and the round shapes of real cavities cause a distortion of multipolar field force lines giving concurrent electric and magnetic longitudinal field components that increase with distance from the axis. Such longitudinal electric components grow linearly in $y$ (to first order) and are caused by the distortion of electric force lines, as demonstrated in Fig. 1 by the $\mathrm{TE}_{111}$ mode. Near the axis, the dipole field is called "hybrid" and is shown in Fig. 2. Figure 3 shows the field distribution of this dipole mode near the LEP cavity axis. All calculated dipole modes have both longitudinal electric and magnetic fields $\left(E_{z}\right.$ and $B_{z}$ ) far from axis.

Although the TE/TM classification is exactly described only for the field patterns of an ideal pillbox cavity, a TE/ TM pillboxlike classification is useful for analyzing real cavities. We will classify these modes as pillboxlike magnetic (TM) and electric (TE) dipole modes according to $[6-8]$.
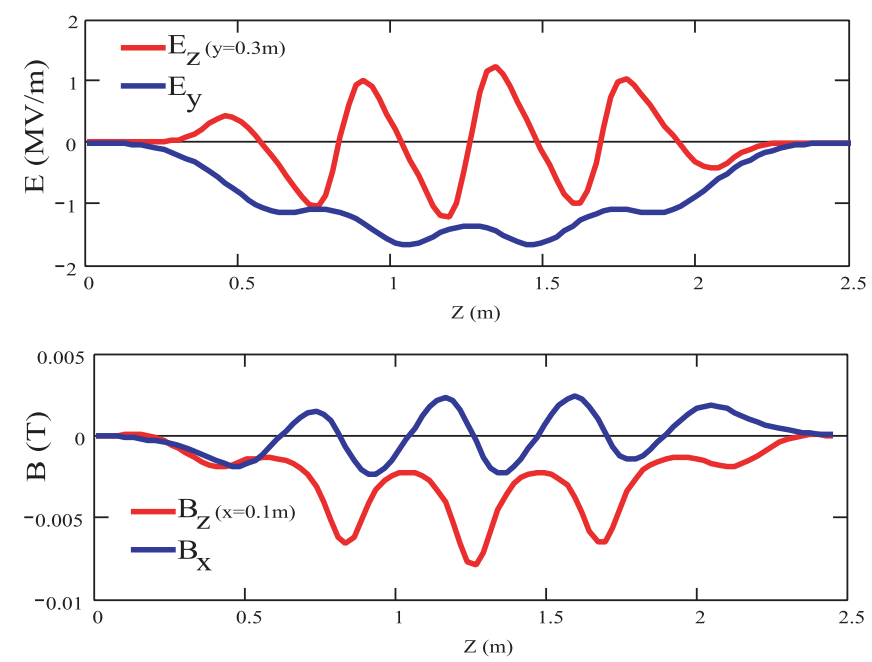

FIG. 3. (Color) Distribution of electromagnetic field amplitudes in the vicinity of the LEP cavity axis for the first dipole mode at $446 \mathrm{MHz}$. Amplitudes correspond to a stored energy of $1 \mathrm{~J} . E_{z}$ and $B_{x}$ amplitudes are linear approximations at the offsets of $y=$ $0.3 \mathrm{~m}$ and $x=0.1 \mathrm{~m}$ correspondingly.

Let us assume that the dipole mode electric field direction is vertical (see Fig. 2). Then the magnetic field will be directed horizontally along the $x$ axis and the rf field will deflect particles vertically. The longitudinal fields are also shown in Fig. 2. To first order approximation, the magnetic field $B_{z}$ increases linearly with the distance from the $x$ axis and the electric field $E_{z}$ is increases linearly in the $y$ direction:

$$
\begin{aligned}
& B_{z}(\bar{r}, t)=B_{z}(x, z, t) \approx x \frac{\partial B_{z}(z)}{\partial x} \sin (\omega t+\varphi), \\
& E_{z}(\bar{r}, t)=E_{z}(y, z, t) \approx y \frac{\partial E_{z}(z)}{\partial y} \cos (\omega t+\varphi) .
\end{aligned}
$$

\begin{tabular}{|c|c|c|c|c|c|c|}
\hline No. & $F[\mathrm{MHz}]$ & Type & $Q_{0}$ & $Q_{\mathrm{ex}}$ & $R,[\mathrm{Ohm} / \mathrm{cm}]$ & $J / B_{\max }{ }^{2}\left[\mathrm{~J} / \mathrm{T}^{2}\right]$ \\
\hline 1 & 446.84 & $\mathrm{TE}_{111}, 0$ & $6.1 \times 10^{9}$ & & 0.038 & 173391 \\
\hline 2 & 453.89 & $\mathrm{TE}_{111}, \pi / 3$ & $6.5 \times 10^{9}$ & & 0.3 & 108672 \\
\hline 3 & 465.36 & $\mathrm{TE}_{111}, 2 \pi / 3$ & $7.1 \times 10^{9}$ & $10400^{\mathrm{a}}$ & 1.7 & 91400 \\
\hline 4 & 479.26 & $\mathrm{TE}_{111}, \pi$ & $8.0 \times 10^{9}$ & $7500^{\mathrm{a}}$ & 0.72 & 54174 \\
\hline 5 & 501.11 & $\mathrm{TM}_{110}, \pi$ & $5.8 \times 10^{9}$ & & 0.35 & 175757 \\
\hline 6 & 509.94 & $\mathrm{TM}_{110}, 2 \pi / 3$ & $6.8 \times 10^{9}$ & $9000^{\mathrm{a}}$ & 2.3 & 70374 \\
\hline 7 & 516.84 & $\mathrm{TM}_{110}, \pi / 3$ & $7.4 \times 10^{9}$ & $21000^{\mathrm{a}}$ & 1.6 & 60298 \\
\hline 8 & 520.23 & $\mathrm{TM}_{110}, 0$ & $7.7 \times 10^{9}$ & & 0.12 & 60770 \\
\hline 9 & 692.14 & & $3.2 \times 10^{9}$ & $7300^{\mathrm{a}}$ & 4.0 & 34449 \\
\hline 10 & 829.21 & & $6.6 \times 10^{9}$ & $2.3 \times 10^{6}$ & 0.0026 & 65520 \\
\hline 11 & 1266.5 & Trapped & $2.3 \times 10^{9}$ & $1.8 \times 10^{5}$ & 0.048 & 8997 \\
\hline 12 & 1498.9 & Trapped & $3.2 \times 10^{9}$ & $6.2 \times 10^{4}$ & 0.013 & 20907 \\
\hline 13 & 1570.1 & Trapped & $3.9 \times 10^{9}$ & $3.1 \times 10^{4}$ & 0.0014 & 115496 \\
\hline 14 & 1627.2 & Trapped & $4.0 \times 10^{9}$ & $1.0 \times 10^{4}$ & 0.0039 & 5413 \\
\hline 15 & 1745.1 & Trapped & $1.5 \times 10^{9}$ & $5.0 \times 10^{4}$ & 0.00055 & 8953 \\
\hline 16 & 1790.0 & Trapped & $2.4 \times 10^{9}$ & $1.7 \times 10^{5}$ & $4.5 \times 10^{-5}$ & 1312021 \\
\hline
\end{tabular}

TABLE I. Performance of the LEP cavity dipole modes.

${ }^{\mathrm{a}}$ Measured in [6] $Q_{\mathrm{ex}}$ is related to the rf power input and HOM couplers. 
Among the calculated modes, the 16 largest external quality factors $\left(Q_{\mathrm{ex}}\right)$ are retained and presented in Table I. While the dipole modes with frequencies over $880 \mathrm{MHz}$ can propagate in a $200 \mathrm{~mm}$ beam pipe, the modes listed in Table I are trapped due to very weak coupling with the beam pipe, which also explains their relatively high $Q_{\text {ex }}$.

As shown by calculations of the Forschungs Zentrum Dresden (FZD) superconducting radio frequency (SRF) gun [9], the uncoupling of trapped dipole modes, as well as a monopole mode [10], from the beam pipe changes sometimes strongly in a resonant manner when the cavity is deformed (compressed or elongated) along the axis. When the coupling coefficient becomes zero, the dipole mode $Q_{\text {ex }}$ reaches its maximum value determined only by losses in the superconducting cavity walls. Therefore, the experimental $Q_{\mathrm{ex}}$ of trapped modes can differ substantially from the calculated values during cavity operation, and reaching the maximum $Q_{\mathrm{ex}}$ is quite probable.

Calculation results presented in Table I indicate that there are several HOMs, which are not measured experimentally [6], namely, at 446, 453, 501, and $520 \mathrm{MHz}$. Most likely, these modes are coupled neither to the rf power input coupler nor to the pickup couplers. Otherwise, they would be measured. If this is indeed the case, these modes can have very high $Q$ of the order of their intrinsic $Q_{0} \sim$ $10^{8}-10^{9}$. These dipole modes are primary candidates for causing the breakup instability and beam lost in the KAERI accelerator because, in the experiments, no excited dipole mode was detected [2].

\section{EXCITATION OF DIPOLE MODES BY BEAM}

Let us assume the beam is initially not displaced at the cavity axis. Excitation of dipole modes by the beam is only possible if the beam is causally (or by dipole mode acting) offset up or down with respect to the axis where the beam transfers its energy to the dipole field by the interaction with longitudinal electric fields. This excitation is proportional to the beam offset (to first order). A weakly relativistic beam is rather easily deflected by the transverse magnetic field of a dipole mode. Therefore, a weakly relativistic beam interacts strongly with dipole modes [11]. Since the resulting offset increases proportionally with mode intensity, the excitation of this mode is proportional to the square of the mode intensity. Instability of dipole mode excitation occurs when the beam excitation power, which is proportional to the beam current, exceeds the power loss of the modes dissipated in the cavity walls; then, such modes can lead to an amplifying action [12]. This problem is especially dramatic in superconducting cavities where there is little power loss.

If the harmonic repetition rate coincides exactly with the resonant frequency of the dipole mode, all bunches arrive at the cavity with the same oscillation (input) phase of the dipole mode. Since only that beam is the excitation source of this mode, the input phase of the oscillation will just be that beam energy loss is maximal. Both dipole HOM and monopole HOM excitations are qualified to this condition. The peculiarity of a dipole excitation is its heavy interconnection with the particle trajectory.

An equivalent scheme, consisting of a parallel resonant circuit driven by a pulsed current source representing the beam bunches, is used to model HOM excitation [7]. The rf voltage $(U)$ across the circuit is equivalent to the energy gain or loss of particles $(\Delta W=e U)$, which is measured in terms of electron volts $(\mathrm{eV})$. For our analyses we use the following properties of the resonant circuits:

$$
Q=\omega \tau / 2, \quad P=\omega J / Q .
$$

Here, $Q$ is the loaded quality factor of a dipole mode; $\omega$ is the angular resonant frequency; $\tau$ is the decay time constant; $P$ is the power loss of a dipole mode with stored electromagnetic energy $J$.

We obtain the energy gain $(U)$ in the dipole mode field by integrating the electric rf field along the particle trajectory inside the cavity $(z=0-L, t=0-T)$ :

$$
\begin{aligned}
U= & \int E_{z}(\bar{r}, t) d z+\int E_{y}(\bar{r}, t) d y \\
= & \int_{0}^{T} y \frac{\partial E_{z}(z)}{\partial y} \cos (\omega t+\varphi) \beta c d t+\int_{0}^{T} y^{\prime} E_{y}(\bar{r}, t) d t \\
= & c B_{\max } \int_{0}^{T} y \frac{\partial E_{z}(z) / c B_{\max }}{\partial y} \cos (\omega t+\varphi) \beta c d t \\
& +c B_{\max } \int_{0}^{T} \frac{E_{y}(z)}{c B_{\max }} y^{\prime} \cos (\omega t+\varphi) d t,
\end{aligned}
$$

where $E_{z}$ is the dipole mode electric field given by Eq. (1), $y^{\prime}=d y / d t$. This integrand is normalized by the maximum amplitude of transverse magnetic field on axis $\left(B_{\max }\right)$; the integral value depends only on the field configuration along the particle trajectory and the input phase $\varphi$.

Figure 4 shows particle trajectories in the $446 \mathrm{MHz}$ dipole field. Bunches with input phases of $-23.6^{\circ}$ and

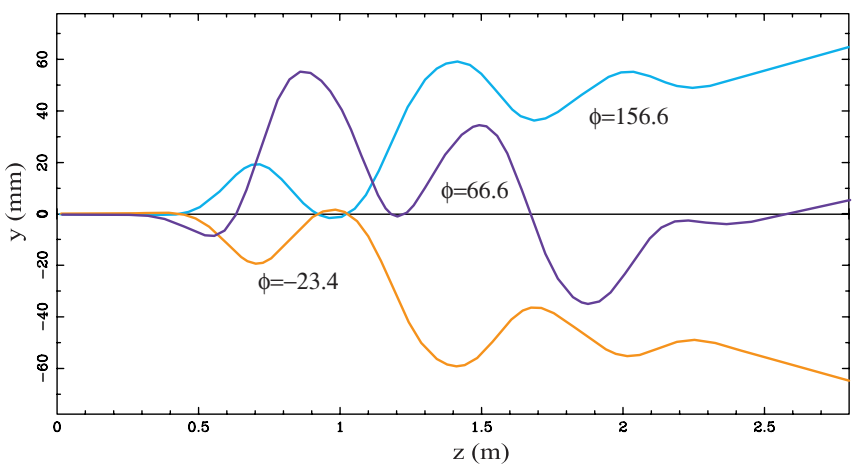

FIG. 4. (Color) Particle trajectories with different input phases $(\varphi)$ in the LEP cavity when the $446 \mathrm{MHz}$ dipole mode with $B_{\max }=0.01 \mathrm{~T}$ and the $352 \mathrm{MHz}$ accelerating mode are excited. 
$-23.6^{\circ}+180^{\circ}=156.6^{\circ}$ have the maximum deflection amplitude at the cavity output, but in opposite directions. All pairs of particles with input phases $\varphi$ and $\varphi+180$ have the same output energies and equal, but opposite, trajectories.

We find the transverse coordinate $y=y(z, t)$ by solving the differential equation for transverse particle motion [13]:

$$
\frac{d}{d t} m \dot{y} \gamma=e E_{y} \cos (\omega t+\varphi)+e \beta c B_{x} \sin (\omega t+\varphi) .
$$

For simplicity we assume, $\beta=$ const, $\gamma=$ const. Normalizing by $B_{\max }$, we get

$$
\begin{aligned}
\ddot{y}= & \frac{e}{m \gamma} c B_{\max }\left[\left(E_{y} / c B_{\max }\right) \cos (\omega t+\varphi)\right. \\
& \left.+\beta\left(B_{x} / B_{\max }\right) \sin (\omega t+\varphi)\right],
\end{aligned}
$$

where the expression in square brackets is dimensionless. Let the solution of Eq. (5) be the equation

$$
\begin{aligned}
y(z, t)= & \frac{e}{m \gamma \omega^{2}} c B_{\max }\left[Y_{1}(z) \cos (\omega t+\varphi)\right. \\
& \left.+Y_{2}(z) \sin (\omega t+\varphi)\right] .
\end{aligned}
$$

If we replace $y$ in Eq. (5) by Eq. (6), we obtain a complex differential equation for the dimensionless function $\dot{Y}=$ $Y_{1}(z)+j Y_{2}(z)$, which depends on dipole field configuration:

$$
\frac{\partial^{2} \dot{Y}}{\partial \psi^{2}}-2 j \frac{\partial \dot{Y}}{\partial \psi}-\dot{Y}=E_{y}(\psi) / c B_{\max }+j \beta B_{x}(\psi) / B_{\max },
$$

where $\psi=k z, k=\omega / \beta c, \beta c=v_{z}$.

Substituting Eq. (6) for $y$ in Eq. (3), we get

$$
U=\frac{e c^{2} B_{\max }^{2}}{2 m \gamma \omega^{2}}\left[I_{1}-I_{2} \cos (2 \varphi-2 \Phi)\right]
$$

where the dimensionless constant $I_{1}$, which depends on dipole field configuration, is given as follows:

$$
\begin{aligned}
I_{1}= & \int_{0}^{k L} \frac{\partial\left(E_{z} / c B_{\max }\right)}{\partial k y} Y_{1}(\psi) d \psi+\int_{0}^{k L}\left(E_{y} / c B_{\max }\right) \\
& \times \frac{\partial Y_{1}(\psi)}{\partial \psi} d \psi+\int_{0}^{k L}\left(E_{y} / c B_{\max }\right) Y_{2}(\psi) d \psi
\end{aligned}
$$

From numerical calculations it follows that the particlefield energy exchanging behavior is similar to Eq. (8) even if $\beta \neq$ const, and furthermore: (i) $U$ depends on twice the input phase $\varphi$ such that replacing the input phase with the opposite phase $\left(\varphi \rightarrow \varphi+180^{\circ}\right)$ does not change anything in the particle-field energy exchange. This is clear since, at these phases, the trajectories are equal and opposite. This means that the resonant excitation of dipole oscillations can be provided by bunches with a repetition frequency twice as high the resonant frequency of the dipole mode.

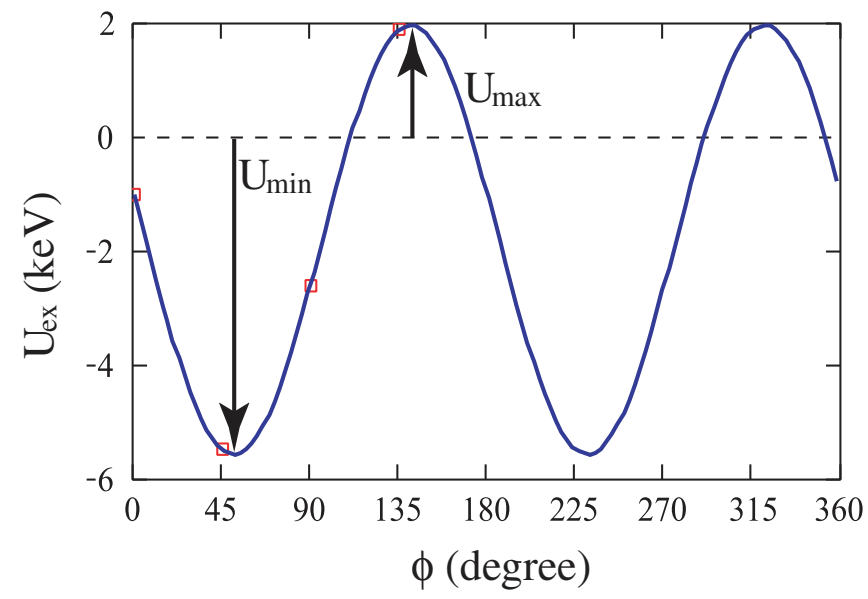

FIG. 5. (Color) Behavior of particle energy exchange with the $465 \mathrm{MHz}$ dipole mode $\left(B_{\max }=0.001 \mathrm{~T}\right)$ versus of the input phase. The particles are accelerated from 2 to $6.33 \mathrm{MeV}$ by the $E_{010}$ mode.

Figure 5 shows a typical particle energy exchange with one dipole mode as a function of the input phase. (ii) In the input phase ranges from $0^{\circ}$ to $360^{\circ}$, the particle energy has two minima and two maxima. Extreme energy variations are proportional to $B_{\max }{ }^{2}$ and differ from each other in magnitude:

$$
\begin{aligned}
& U_{\min }=\frac{e c^{2} B_{\max }^{2}}{2 m \gamma \omega^{2}}\left(I_{1}-\left|I_{2}\right|\right), \\
& U_{\max }=\frac{e c^{2} B_{\max }^{2}}{2 m \gamma \omega^{2}}\left(I_{1}+\left|I_{2}\right|\right) .
\end{aligned}
$$

Table II shows these values for the dipole modes calculated up to $2 \mathrm{GHz}$. (iii) As mentioned above, for resonant excitation, the dipole mode phase is the phase when the particle loses maximum energy $U_{\text {ex }}=U_{\min }<0$, i.e. $\varphi=\Phi$ or $\varphi=\Phi+180^{\circ}$. One of the two equivalent phases is randomly selected.

Let us now analyze data from Table II for cases when bunches are not resonant with dipole HOMs (the resonant frequency is not a multiple of the bunch repetition rate). In such a case, the input phase differs from bunch to bunch by $\Delta \varphi=2 \pi \omega / n \Omega$. Here, we assumed that the dipole mode oscillation frequency excited by each bunch is exactly equal to the resonance frequency of the cavity dipole mode. In Ref. [10], the formula was derived for a vector sum voltage excited by such bunches. It has been shown that the voltage has resonant dependence (with a $Q$ factor) on the bunch repetition rate harmonics $(n \Omega)$. For the high $Q$ factors of a superconducting cavity, the probability of the repetition rate harmonic to be within the small resonance band of a dipole mode is negligible. If by chance the repetition rate harmonic gets into the band of the dipole mode resonance, the problem can be overcome by using another repetition frequency, e.g., $(n \pm 1) \Omega$. Thus, in reality, the energy lost by the beam to the superconducting 
TABLE II. Performance of the dipole mode excitation by a weakly relativistic beam in the LEP cavity. Particles with initial energy of $2 \mathrm{MeV}$ are accelerated by the $\mathrm{TM}_{010}$ mode up to $6.35 \mathrm{MeV}$. $B_{\max }=0.001 \mathrm{~T}$ is the maximum field of dipole modes. $I_{\mathrm{thr}}$ is the breakup instability threshold current discussed in the next section, $Q \sim Q_{\mathrm{ex}}$ are taken from Table I.

\begin{tabular}{lccccccc}
\hline \hline$N$ & $F[\mathrm{MHz}]$ & $\Phi[$ degree $]$ & $U_{\min }[\mathrm{eV}]$ & $U_{\max }[\mathrm{eV}]$ & $U_{\mathrm{ex}}[\mathrm{eV}]$ & $I_{\text {thr }} Q[\mathrm{~A}]$ & $I_{\text {thr }}[\mathrm{A}]$ \\
\hline 1 & 446.8 & 44 & -92.9 & 494 & 200 & $\infty$ & $\infty$ \\
2 & 453.9 & 149 & -3074 & -1210 & -2141 & $1.4 \times 10^{5}$ & $>2.2 \times 10^{-5}$ \\
3 & 465.4 & 53 & -5840 & 1793 & -2024 & $1.3 \times 10^{5}$ & 12.5 \\
4 & 479.3 & 126 & 926 & 1840 & 1383 & $\infty$ & $\infty$ \\
5 & 501.1 & 16 & 1710 & 9284 & 5497 & $\infty$ & $\infty$ \\
6 & 509.9 & 82 & 2060 & 9770 & 5914 & $\infty$ & $\infty$ \\
7 & 516.8 & 154 & -2440 & 2795 & 178 & $\infty$ & $\infty$ \\
8 & 520.2 & 50 & -1090 & -78 & -583 & $3.4 \times 10^{5}$ & $>4.4 \times 10^{-5}$ \\
9 & 692.1 & 126 & -247 & 1500 & 629 & $\infty$ & $\infty$ \\
10 & 829.2 & 55 & -61.8 & -31. & -46.3 & $7.4 \times 10^{6}$ & 3.20 \\
11 & 1266.5 & 65 & -5.3 & 17.3 & 6.02 & $\infty$ & $\infty$ \\
12 & 1498.9 & 22 & -11.6 & 27.1 & 7.7 & $\infty$ & $\infty$ \\
13 & 1570.1 & 78 & -25.5 & -5.7 & -15.9 & $7.2 \times 10^{7}$ & $2.3 \times 10^{3}$ \\
14 & 1627.2 & 160 & -1.04 & 8.03 & 3.5 & $\infty$ & $\infty$ \\
15 & 1745.1 & 37 & -2.68 & 7.1 & 2.2 & $\infty$ & $\infty$ \\
16 & 1790.0 & 132 & -36.6 & 24.9 & -5.9 & $2.5 \times 10^{9}$ & $1.5 \times 10^{4}$ \\
\hline \hline
\end{tabular}

cavities should be calculated as an integral of Eq. (8) over all phases $\varphi=0-2 \pi$, which gives

$$
U_{\mathrm{ex}}=\left(U_{\max }+U_{\min }\right) / 2,
$$

i.e., one must set $I_{2}=0$ in Eq. (8).

There are modes that can never be excited $\left(U_{\mathrm{ex}}>0\right)$. The other modes can be excited. The necessary instability condition is $U_{\mathrm{ex}}<0$, when particles transfer a part of their energy to the dipole mode. This corresponds to the case shown in Fig. 5, where the average exchanged energy is negative.

A continuous beam can excite these "negative" dipole modes. This follows from the principle of superposition. The continuous beam can be represented by a continuous succession of short bunches; each of which independently excites the dipole mode. The energy given by such a beam is calculated as a sum of the individual bunch's energies. An ordinary whistle by continuous airflow is an example of such oscillations [2].

\section{BREAKUP INSTABILITY THRESHOLD CURRENT}

As was mentioned above, in order to satisfy the instability condition, the power loss in the cavity walls $(P=$ $\omega J / Q)$ should be less than or equal to the power transferred by the beam $\left(P_{\mathrm{ex}}\right): P_{\mathrm{ex}}=U_{\mathrm{ex}} \cdot 2 I_{0} / 2$, where $2 I_{0}$ is the amplitude of the first harmonic of the pulsed beam current with an average current $I_{0}$. Thus, the necessary and sufficient conditions for the breakup instability caused by a dipole mode are

$$
U_{\text {ex }}<0 \quad\left|U_{\text {ex }}\right| I_{0} \geq \omega J / Q .
$$

The instability threshold current $I_{\text {thr }}$ is obtained from Eq. (12) when two sides of the equation are equal. If we combine Eq. (12) with Eq. (8), we get

$$
I_{\mathrm{thr}} Q=\omega \frac{J / B_{\max }^{2}}{\left|U_{\mathrm{ex}}\right| / B_{\max }^{2}}=\frac{2 m \gamma c^{2}}{e\left|I_{1}\right|} \omega^{3}\left(J / B_{\max }^{2}\right)
$$

The averaging $\left(I_{2}=0\right)$ is taken into account in Eq. (13).

The instability threshold currents calculated for each dipole mode $\left(I_{\mathrm{thr}} Q\right.$ and $\left.I_{\mathrm{thr}}\right)$ are presented in Table II. We used the characteristics of the dipole modes $\left(J / B_{\max }^{2}\right), Q$ factors, and excitation voltages $\left(U_{\mathrm{ex}}\right)$ given both by calculations and experiments of [6] from Tables I and II.

Although the threshold current equation (13) is proportional to $\omega^{3}$, the frequency increasing of a fundamental mode by scaling of cavity sizes to increase all dipole mode frequencies is not perspective. This is due to the proportionality of the mode field energy $\left(J / B_{\max }{ }^{2}\right)$ to the cavity volume, which is inversely proportional to the cube of the fundamental mode frequency.

Let us now study the role of the initial beam trajectory offset $\left(Y_{o}=\left.y\right|_{z=0}\right)$ and slope $\left(Y^{\prime}{ }_{o}=\partial y /\left.\partial z\right|_{z=0}\right)$. For this, let the solution of Eq. (5) be given by

$$
\begin{aligned}
y(z, t)= & \frac{e}{m \gamma \omega^{2}} c B_{\max }\left[Y_{1}(z) \cos (\omega t+\varphi)\right. \\
& \left.+Y_{2}(z) \sin (\omega t+\varphi)\right]+Y_{o}+Y_{o}^{\prime} \cdot z
\end{aligned}
$$

It can be shown that $Y_{1}(z)$ and $Y_{2}(z)$ are solutions of Eq. (7). 
If we replace $y$ by Eq. (14) in Eq. (3), we obtain

$$
U=\frac{e c^{2} B_{\max }^{2}}{2 m \gamma \omega^{2}}\left[I_{1}-I_{2} \cos (2 \varphi-2 \Phi)-I_{o} \cos (\varphi-\Phi)\right]
$$

In nonresonant cases, integration of Eq. (15) in the range of $\varphi=0-2 \pi$ gives the same $U_{\mathrm{ex}}$ as Eq. (11). Therefore, the threshold current of beam breakup instability does not depend on the initial beam trajectory offset and slope (within limits of dipole field linear approximation near the axis). This fact is clearly proven by numerical calculations and explains the threshold current independence from beam trajectory offsets and slopes found experimentally [2].

To conclude this section, let us summarize.

There are two classes of dipole modes: (i) First class: modes which are always stable and have $U_{\mathrm{ex}}>0$. These modes can be ignored in accelerator operation since they rarely affect beam stability. (ii) Second class: modes having $U_{\mathrm{ex}}<0$. These dipole modes are primary candidates for causing breakup instability at low average beam current.

From Eq. (13) it follows that the threshold current increases as the cube of the dipole mode frequency. This is proven by the numerical calculations (see Table II). Therefore, all dipole modes with high resonance frequencies can also be discounted in the accelerator operation. These higher frequency dipole modes are mostly trapped for the case of LEP cavity considered, as shown in Table I.

The most dangerous dipolar modes are those at 453 and $520 \mathrm{MHz}$. If the loaded $Q$ factors of these modes are on the order of $10^{8}$, the corresponding threshold currents are just a few milliamperes, which explains the values found experimentally [2]. These modes are not trapped and, therefore, it is possible to decrease their $Q$ factors. To improve coupling of these modes to the beam pipe loads, fluted beam pipes, as in Ref. [14], can be used. A different method of increas- ing the threshold beam current is proposed in the next section. It is especially effective for the case of high- $Q$ trapped modes in multicell superconducting cavities.

\section{SUPPRESSION OF BREAKUP INSTABILITY BY RF FOCUSING}

Using transversal beam focusing inside the cavity decreases displacement of a particle from the cavity axis. Then, the interaction of the particle with the longitudinal field of the dipole mode becomes weaker as the field is smaller near the axis. Thus, the focusing should increase the threshold current of breakup instability. This method was first described in Ref. [12]. Since the solenoid field cannot be used in superconducting cavities, we propose to use $\mathrm{rf}$ focusing by higher-order magnetic modes of $\mathrm{TE}_{\mathrm{onm}}$ type. We will refer to these modes as "TE modes" below.

TE modes have longitudinal magnetic rf field on the cavity axis. The rf electric field has an azimuthal direction around the axis. In superconducting cavities, the maximum rf magnetic field on axis can reach $0.3 \mathrm{~T}$. In this case, the magnetic field at the cavity walls does not exceed the critical value of superconductivity break down $(0.2 \mathrm{~T})$. The use of $\mathrm{rf}$ focusing by higher-order TE magnetic modes in superconducting cavities of rf guns was studied in Refs. [15,16]. Particular analysis and applications of $\mathrm{rf}$ focusing are considered in [17].

The rf focusing force dependence on field intensity and particle energy is the same as that for solenoids $\sim\left(B_{\mathrm{TE}} / \beta \gamma\right)^{2}$. The only difference is that the rf lens has a small variable component of the focusing force with the double the frequency $\left(2 \omega_{\mathrm{TE}}\right)$, whose amplitude decreases exponentially with TE-mode frequency $[15,17]$. If we take a TE mode with sufficiently high frequency, this variable part of the focusing force may be neglected.

In the LEP cavity, there are 40 TE-type HOMs below the cutoff frequency of the cylindrical beam pipe $(1820 \mathrm{MHz})$, (see Table III). In principle, any TE mode can be used. We

TABLE III. Performance of $\mathrm{TE}_{\text {onm }}$-type HOMs of the LEP cavity applicable for $\mathrm{rf}$ focusing.

\begin{tabular}{|c|c|c|c|c|c|c|c|c|}
\hline$N$ & $F[\mathrm{MHz}]$ & $Q_{0}$ & No. & $F[\mathrm{MHz}]$ & $Q_{0}$ & No. & $F[\mathrm{MHz}]$ & $Q_{0}$ \\
\hline 1 & 667.78 & $7.02 \times 10^{9}$ & 14 & 1278.9 & $3.48 \times 10^{9}$ & 27 & 1548.9 & $2.79 \times 10^{9}$ \\
\hline 2 & 668.31 & $7.11 \times 10^{9}$ & 15 & 1305.0 & $2.85 \times 10^{9}$ & 28 & 1555.3 & $2.79 \times 10^{9}$ \\
\hline 3 & 669.12 & $7.1 \times 10^{9}$ & 16 & 1311.8 & $3.24 \times 10^{9}$ & 29 & 1618.9 & $3.66 \times 10^{9}$ \\
\hline 4 & 994.59 & $3.8 \times 10^{9}$ & 17 & 1335.0 & $3.26 \times 10^{9}$ & 30 & 1621.2 & $3.83 \times 10^{9}$ \\
\hline 5 & 995.39 & $3.9 \times 10^{9}$ & 18 & 1352.7 & $3.41 \times 10^{9}$ & 31 & 1638.3 & $3.02 \times 10^{9}$ \\
\hline 6 & 997.67 & $4 \times 10^{9}$ & 19 & 1374.0 & $2.66 \times 10^{9}$ & 32 & 1676.8 & $3.05 \times 10^{9}$ \\
\hline 7 & 1000.7 & $4.12 \times 10^{9}$ & 20 & 1374.4 & $2.71 \times 10^{9}$ & 33 & 1685.1 & $3.02 \times 10^{9}$ \\
\hline 8 & 1013.9 & $5.36 \times 10^{9}$ & 21 & 1386.3 & $3.67 \times 10^{9}$ & 34 & 1698.0 & $3.03 \times 10^{9}$ \\
\hline 9 & 1015.2 & $5.44 \times 10^{9}$ & 22 & 1387.4 & $3.81 \times 10^{9}$ & 35 & 1714.1 & $2.41 \times 10^{9}$ \\
\hline 10 & 1017.1 & $5.61 \times 10^{9}$ & 23 & 1389.8 & $3.75 \times 10^{9}$ & 36 & 1734.1 & $2.87 \times 10^{9}$ \\
\hline 11 & 1018.2 & $5.78 \times 10^{9}$ & 24 & 1482.2 & $2.9 \times 10^{9}$ & 37 & 1747.3 & $3.01 \times 10^{9}$ \\
\hline 12 & 1255.8 & $3.17 \times 10^{9}$ & 25 & 1509.7 & $2.57 \times 10^{9}$ & 38 & 1755.3 & $3.24 \times 10^{9}$ \\
\hline 13 & 1268.9 & $3.31 \times 10^{9}$ & 26 & 1537.5 & $2.78 \times 10^{9}$ & 39 & 1758.8 & $3.4 \times 10^{9}$ \\
\hline
\end{tabular}




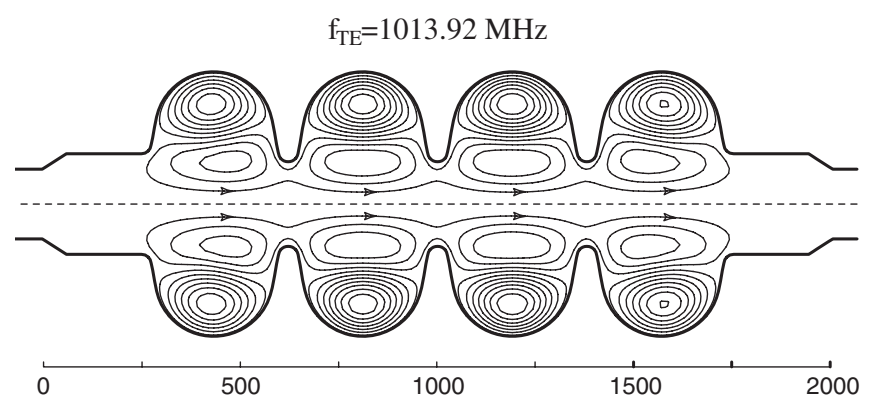

FIG. 6. Magnetic field pattern of the focusing mode $\mathrm{TE}_{021}(0-$ type).

have selected the $1013.9 \mathrm{MHz} \mathrm{TE}_{021}$ 0-type mode. For this mode, the ratio of the peak magnetic field at the surface $\left(B_{\text {peak }}\right)$ to the maximum field on axis $\left(B_{\mathrm{TE}}\right)$ is relatively small: $B_{\text {peak }} / B_{\mathrm{TE}}=0.46$. Figure 6 shows the magnetic rf field pattern of this TE mode in the cavity.

To study the effect of focusing by 1013.9 MHz TE mode on the energy exchange between particles and dipole modes, the dynamics of bunches in the LEP cavity were modeled numerically. The maximum axial magnetic field of TE mode was varied in the range of $B_{\mathrm{TE}}=0-0.3 \mathrm{~T}$. Calculations were performed for four input phases $\left(0^{\circ}\right.$, $\left.45^{\circ}, 90^{\circ}, 135^{\circ}\right)$. Then, $U_{\text {ex }}$ were calculated by integrating Eq. (3) along the trajectories calculated by ASTRA. Upon averaging $U_{\mathrm{ex}}$ over these four phases for each dipole mode, the threshold currents of the beam breakup instability were calculated using Eq. (13), which takes into account the influence of the TE-mode focusing and acceleration by $\mathrm{TM}_{010}$ mode $(E=2 \mathrm{MeV} \rightarrow 6.35 \mathrm{MeV})$.

Figure 7 shows trajectories of bunch in the cavity at various input phases of the $465 \mathrm{MHz}$ dipole mode for the maximum magnetic field of $0.1 \mathrm{~T}$ for the $1013 \mathrm{MHz}$ TE mode on axis. The deviation of the bunches with respect to the axis is substantially smaller than for the case without focusing by the TE mode $\left(B_{\mathrm{TE}}=0\right)$.

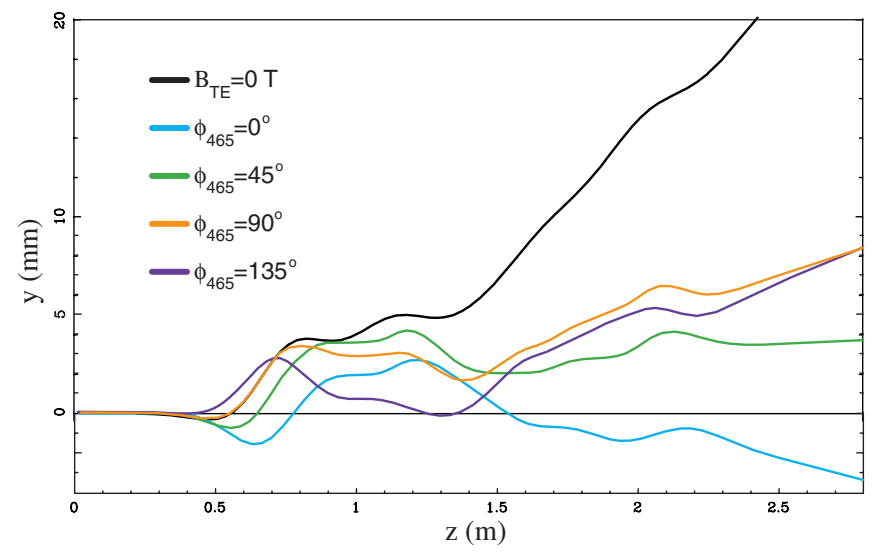

FIG. 7. (Color) Bunch trajectories in LEP cavity excited by three rf fields: $\mathrm{TM}_{010} 352 \mathrm{MHz}\left(E_{\max }=5 \mathrm{MV} / \mathrm{m}\right)$, dipole $\mathrm{TE}_{111}$ $465 \mathrm{MHz}\left(B_{\max }=0.01 \mathrm{~T}\right)$, and $\mathrm{TE}_{021} 1013 \mathrm{MHz}\left(B_{\mathrm{TE}}=0.1 \mathrm{~T}\right)$.
Phase dependence of the energy exchange between particles and the dipole mode field follows twice the frequency, as that is the case without magnetic focusing mode (see Fig. 5). In addition to numerical calculation, this fact can be derived analytically. In order to take into account the influence of the focusing force of the magnetic mode, it is sufficient to add to Eq. (4) the term of TE focusing mode force [17] in the form of $-\left[e B_{\mathrm{TE}}(z) / 2 m \gamma\right]^{2} y$, where only the constant component is taken into account. New differential equation for trajectories $Y_{1}(z)$ and $Y_{2}(z)$ will differ from the previous equation (13) only by a larger coefficient at $Y(z)$ or by larger "betatron" frequency:

$$
\begin{gathered}
\frac{\partial^{2} \dot{Y}}{\partial \psi^{2}}-2 j \frac{\partial \dot{Y}}{\partial \psi}-\dot{Y}\left[1+\left(\frac{e B_{\mathrm{TE}}}{2 m \omega \gamma}\right)^{2}\right] \\
=E_{y}(\psi) / c B_{\max }+j \beta B_{x}(\psi) / B_{\max }
\end{gathered}
$$

here $B_{\mathrm{TE}}=B_{\mathrm{TE}}(z)$ is the distribution of the TE-mode field along the axis. Therefore Eqs. (8) and (9) for the dipole mode energy exchange with particles do not change.

Numerical calculations (Fig. 8) show that the energy transferred by particles to the field of dipole mode $U_{\mathrm{ex}}=$ $\left(U_{\min }+U_{\max }\right) / 2$ mainly decreases with increasing focusing strength of the TE mode. For the $1013 \mathrm{MHz}^{\mathrm{T}} \mathrm{E}_{021}$ mode with the maximum field on axis greater than $0.133 \mathrm{~T}$, the $465 \mathrm{MHz}$ dipole mode could not be instable since particles do not lose energy $\left(U_{\text {ex }}>0\right)$ to this dipole mode. Modes at 453 and 520 cease to be instable at focusing mode fields greater than 0.22 and $0.285 \mathrm{~T}$, re-

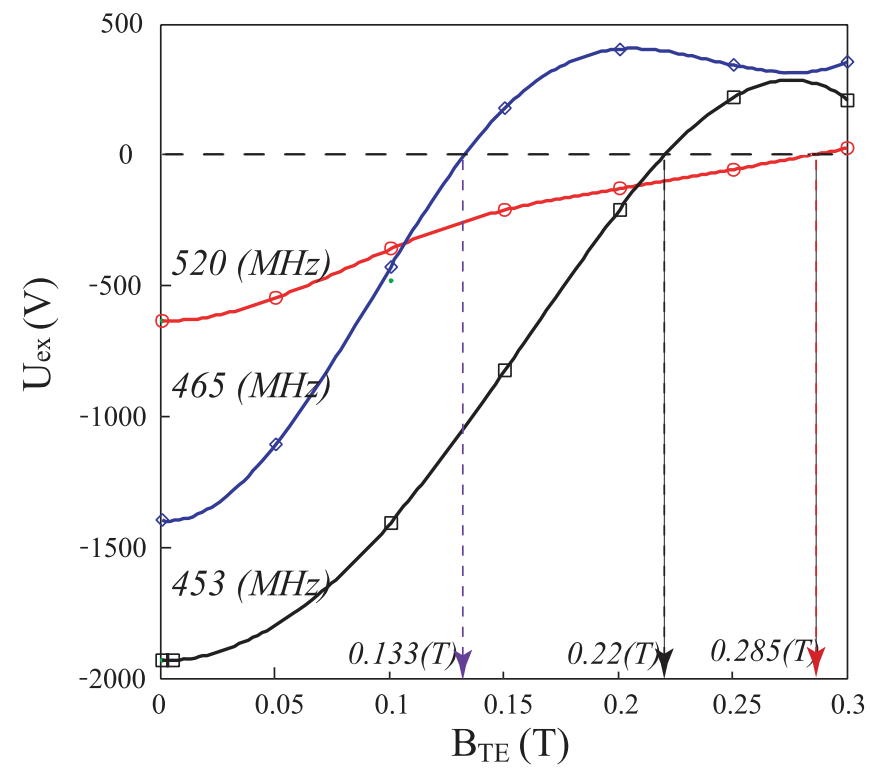

FIG. 8. (Color) Particle-dipole mode energy exchange behavior versus focusing strength of the $1013 \mathrm{MHz} \mathrm{TE}_{021}$ mode $\left(B_{\mathrm{TE}}\right.$ is the maximum TE magnetic field on-axis). The particles are accelerated from 2 to $6.35 \mathrm{MeV}$ by the fundamental $\mathrm{TM}_{010}$ mode. 


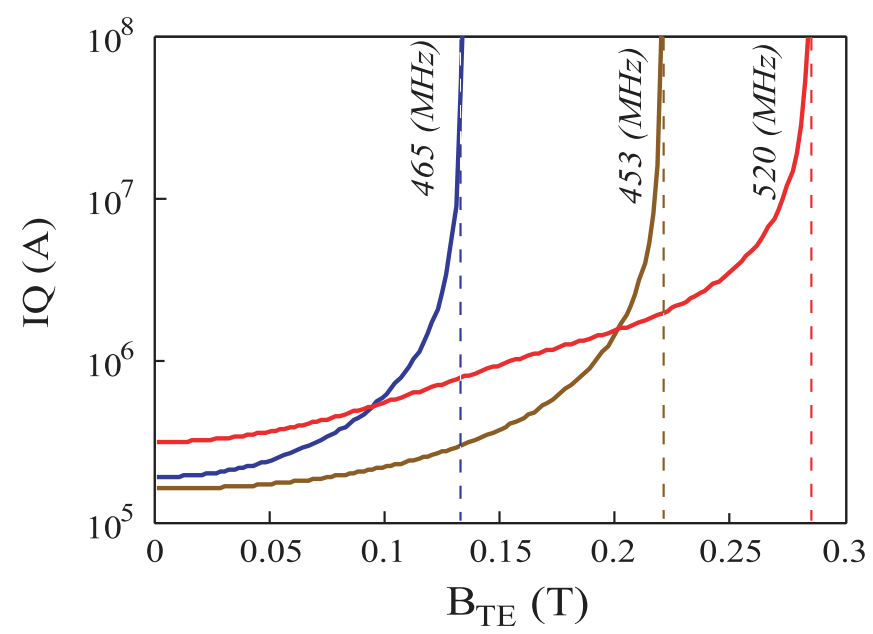

FIG. 9. (Color) Threshold currents of the breakup instability due to the 453,465 , and $520 \mathrm{MHz}$ dipole modes depending on the $1013 \mathrm{MHz} \mathrm{TE}_{021}$ mode magnetic field magnitude.

spectively. The threshold current $\left(I_{\mathrm{thr}} Q\right)$ for the instabilities of the three aforementioned dipole modes increases with the strength of $\mathrm{rf}$ focusing as the beam becomes stable as shown in Fig. 9.

This result demonstrates that the use of TE-mode focusing very effectively suppresses instability due to all three considered dipole modes. Breakup instability due to other dipole modes is also well suppressed. Table IV shows results of calculations for all considered dipole modes of the LEP cavity.

Unfortunately, the maximum surface magnetic field achievable in the LEP cavities is too low to use TE-mode focusing because of the properties of niobium sputtered on a copper substrate, which the cavities are made from. For the fundamental mode at operating gradients (field on axis $E_{\max }=5 \mathrm{MV} / \mathrm{m}$ ), the maximum field on the surface is $B_{\text {peak }} \sim 0.01 \mathrm{~T}$. The magnetic field required to suppress the beam breakup instability by focusing with $\mathrm{TE}_{021}$ mode is $B_{\text {peak }}>0.05 \mathrm{~T}\left(B_{\mathrm{TE}}>0.1 \mathrm{~T}\right)$, which is too high relative to the fundamental mode. Therefore, some other technique will have to be used.

It would be very interesting to study how TE modes affect the beam parameters (emittance, transversal size of bunches, etc.) in order to select the most efficient TE mode, etc., despite the fact that it is known that rf focusing improves the beam characteristics $[16,17]$. However, this will be the object of another article.

In multicell superconducting cavities, the number of HOMs, including trapped ones, grows proportionally with the cell number. As was mentioned above, trapped dipole modes can have very high $Q$ factors emergent during the operation. Therefore, applying the rf focusing to suppress the breakup instability in nine-cell TESLA-like or in other multicell superconducting cavities is important to get high beam currents. To obtain a maximum focusing effect, TE modes of 0 or $\pi$ type must be applied with a flattop distribution in the cavity similar to the fundamental mode. Simulations predict that this is possible by making a special shape at the end cells. Such cavities must be disposed at the beginning of an accelerator, where the particle energy is relatively low.

This technique is especially important for rf guns based on multicell superconducting cavities like FZD [18] because its beam energy starts from zero at the photocathode.

TABLE IV. Breakup instability threshold currents multiplied by $Q$ factor $\left(I_{\mathrm{thr}} Q[\mathrm{~A}]\right)$ for all considered dipole modes of the LEP cavity for different values of the maximum $\mathrm{TE}_{021}$ mode magnetic field $\left(B_{\mathrm{TE}}\right)$ on the cavity axis. Blank spaces are corresponded to infinite values.

\begin{tabular}{|c|c|c|c|c|c|}
\hline \multirow[t]{2}{*}{$N$} & \multirow{2}{*}{$\begin{array}{c}F \\
{[\mathrm{MHz}]}\end{array}$} & \multicolumn{4}{|c|}{$B_{\mathrm{TE}}[\mathrm{T}]$} \\
\hline & & 0 & 0.1 & 0.2 & 0.3 \\
\hline 1 & 446.8 & & $5.4 \times 10^{6}$ & $1.3 \times 10^{6}$ & \\
\hline 2 & 453.9 & $1.4 \times 10^{5}$ & $1.9 \times 10^{5}$ & $4.5 \times 10^{6}$ & \\
\hline 3 & 465.4 & $1.3 \times 10^{5}$ & $4.7 \times 10^{5}$ & & \\
\hline 4 & 479.3 & & & & \\
\hline 5 & 501.1 & & & & \\
\hline 6 & 509.9 & & & & \\
\hline 7 & 516.8 & & $1.9 \times 10^{6}$ & & \\
\hline 8 & 520.2 & $3.4 \times 10^{5}$ & $6.7 \times 10^{5}$ & $2.4 \times 10^{6}$ & \\
\hline 9 & 692.1 & & & $1.6 \times 10^{6}$ & \\
\hline 10 & 829.2 & $7.4 \times 10^{6}$ & & & \\
\hline 11 & 1267 & & $6.7 \times 10^{7}$ & & \\
\hline 12 & 1499 & & & $2.1 \times 10^{7}$ & $9.2 \times 10^{6}$ \\
\hline 13 & 1570 & $7.2 \times 10^{7}$ & & & \\
\hline 14 & 1627 & & $1.2 \times 10^{7}$ & & $6.5 \times 10^{6}$ \\
\hline 15 & 1745 & & & & \\
\hline 16 & 1790 & $2.5 \times 10^{9}$ & $1.4 \times 10^{9}$ & $2.8 \times 10^{8}$ & \\
\hline
\end{tabular}




\section{CONCLUSION}

There are two classes of dipole modes: those that never breakup the beam and those that always provoke the breakup instability even for a continuous beam at a current exceeding some threshold. The threshold current value grows proportionally with the cube of the dipole mode frequency and proportionally with the full particle energy. Threshold current values are independent of initial beam trajectory offsets and angles within reasonable limits.

The possibility of suppressing the breakup instability using rf focusing with the $\mathrm{TE}_{021}$ mode is discussed. This technique is especially important for multicell superconducting cavities having many trapped dipole modes that can have very high $Q$ factors. For example, in TESLA-like nine-cell cavities or in superconducting rf guns, like FZD [18].

\section{ACKNOWLEDGMENTS}

The author wishes to thank his colleagues G. Ya. Kurkin and S. Miginsky for useful scientific discussions and providing experimental data from KAERI, to V. M. Petrov and other colleagues from BINP for interest in this work. The author is especially thankful for the important comments and corrections from S. Belomestnykh at Cornell University.

[1] V.S. Arbuzov, E. I. Gorniker, Yu. A. Evtushenko, E. K. Kendjebulatov, E.I. Kolobanov, A. A. Kondakov, S. A. Krutikhin, I. V. Kuptsov, G. Ya. Kurkin, L. E. Medvedev, S. V. Motygin, V. N. Osipov, V. M. Petrov, A. M. Pilan, A. M. Popov, I. K. Sedlyarov, and A. G. Tribendis, RF system for the industrial linear electron accelerator at KAERI (Daejeon, Korea). RuPAC XIX, Dubna 2004.

[2] G. Ya. Kurkin and S. Miginsky (private communication).
[3] D. G. Myakishev and V. P. Yakovlev, in Proceedings of the 18th Particle Accelerator Conference, New York, 1999 (IEEE, New York, 1999), pp. 2775-2777.

[4] K. Floettmann, ASTRA User Manual, available on the internet: http://www.desy.de/ mpyflo/Astra dokumentation.

[5] G. Arnolds-Mayer, C. Benvenuti, Ph. Bernard, D. Bloess, G. Cavallari, E. Chiaveri, W. Erdt, E. Haebel, N. Hilleret, P. Legendre, H. Lengeler, G. Passardi, J. Schmid, R. Stierlin, J. Tuckmantel, and W. Weingarten, in Proceedings of the Third Workshop on RF Superconductivity (Argonne National Laboratory, Argonne, 1988), Vol. I, pp. 55-79.

[6] E. Haebel, Report No. CERN/RF 87-4, 1987.

[7] T. Wangler, RF Linear Accelerators (Wiley, New York, 1998).

[8] H. Padamsee, J. Knobloch, and T. Hays, RF Superconductivity for Accelerators (Wiley, New York, 1998).

[9] V. Volkov and D. Janssen, (unpublished).

[10] V. Volkov and D. Janssen, in Proceedings of the FEL Conference 2007, Novosibirsk, Russia, see http:// accelconf.web.cern.ch/AccelConf/f07/INDEX.HTM.

[11] V. K. Neil and R. K. Cooper, Part. Accel. 1, 111 (1970).

[12] W. K. H. Panofsky and M. Bander, Rev. Sci. Instrum. 39, 206 (1968).

[13] J.D. Jackson, Classical Electrodynamics (Wiley, New York, 1998), 3rd ed.

[14] H. Padamsee et al., Part. Accel. 40, 17 (1992).

[15] V. Volkov, Ph.D. thesis, Novosibirsk, 2007.

[16] V. Volkov, K. Floettmann, and D. Janssen, in Proceedings of the 2007 Particle Accelerator Conference, Albuquerque, New Mexico, 2007 (IEEE, Albuquerque, New Mexico, 2007).

[17] V. Volkov and D. Janssen, Phys. Rev. ST Accel. Beams 11, 061302 (2008).

[18] D. Janssen et al., in the 32nd Advanced ICFA Beam Dynamics Workshop on Energy Recovering Linacs ERL 2005, Jefferson Laboratory, Newport News, 2005. 